\title{
Automatic selection of indicators in a fully saturated regression
}

\author{
David F. Hendry • Søren Johansen · Carlos Santos
}

Published online: 4 April 2008

(C) Springer-Verlag 2008

\section{Erratum to: Computational Statistics DOI 10.1007/s00180-007-0054-z}

The order of the authors should be

David F. Hendry · Søren Johansen · Carlos Santos

The paper contains some misprints and to avoid misunderstandings we choose to present here the main results again.

Theorem 1 Let $y_{t}=\mu+\sigma_{\varepsilon} \varepsilon_{t}, t=1, \ldots, T$ be i.i.d., where $\varepsilon_{t}$ has symmetric continuous density $f$ (.) with mean zero, variance one, and $E\left[\varepsilon_{t}^{8}\right]<\infty$. Let $T=T_{1}+T_{2}$, and assume that $T_{1} / T \rightarrow \lambda_{1}$ and $T_{2} / T \rightarrow \lambda_{2}$ where $0<\lambda_{1}, \lambda_{2}<1$, with $\lambda_{1}+\lambda_{2}=1$,

The online version of the original article can be found under doi:10.1007/s00180-007-0054-z.

Financial Support from the UK Economic and Social Research Council under a professorial Research Fellowship, RES 051270035 and grant RES 000 230539; continuing support of the Danish Social Sciences Research Council; and funding from the Fundação para Ciência e a Tecnologia (Lisboa) are gratefully acknowledged by D. F. Hendry, S. Johansen and C. Santos respectively.

\section{F. Hendry}

Department of Economics, University of Oxford, Oxford, UK

S. Johansen

Department of Economics, University of Copenhagen, Copenhagen, Denmark

S. Johansen

CREATES, Aarhus, Denmark

C. Santos $(\varangle)$

Department of Economics and Management, Portuguese Catholic University,

Rua Diogo Botelho 1327, 4169-005 Porto, Portugal

e-mail: csantos@porto.ucp.pt 
then the limit distribution of the estimator $\widetilde{\mu}$, see (5), is given by:

$$
T^{1 / 2}(\widetilde{\mu}-\mu) \stackrel{\mathrm{D}}{\rightarrow} \mathrm{N}\left[0, \sigma_{\varepsilon}^{2} \sigma_{\mu}^{2}\right]
$$

where

$$
\sigma_{\mu}^{2}=\left(\int_{-c_{\alpha}}^{c_{\alpha}} f(\varepsilon) d \varepsilon\right)^{-2}\left[\int_{-c_{\alpha}}^{c_{\alpha}} \varepsilon^{2} f(\varepsilon) d \varepsilon\left(1+4 c_{\alpha} f\left(c_{\alpha}\right)\right)+\left(\frac{\lambda_{1}^{2}}{\lambda_{2}}+\frac{\lambda_{2}^{2}}{\lambda_{1}}\right)\left(2 c_{\alpha} f\left(c_{\alpha}\right)\right)^{2}\right]
$$

Note that $\int_{-c_{\alpha}}^{c_{\alpha}} f(\varepsilon) d \varepsilon=1-\alpha$, and for the normal distribution, $f(\varepsilon)=\phi(\varepsilon)$, we find the expression:

$$
\int_{-c}^{c} \varepsilon^{2} \phi(\varepsilon) d \varepsilon=\int_{-c}^{c} \phi(\varepsilon) d \varepsilon-2 c \phi(c)
$$

so that:

$\sigma_{\mu}^{2}=\frac{1}{(1-\alpha)}\left(1+4 c_{\alpha} \phi\left(c_{\alpha}\right)-\frac{2 c_{\alpha} \phi\left(c_{\alpha}\right)}{(1-\alpha)}\left[1+2 c_{\alpha} \phi\left(c_{\alpha}\right)\left(2-\left(\frac{\lambda_{1}^{2}}{\lambda_{2}}+\frac{\lambda_{2}^{2}}{\lambda_{1}}\right)\right)\right]\right)$

Theorem 2 Under the assumptions of Theorem 1 it holds that the estimator $\widetilde{\sigma}_{\varepsilon}^{2}$, see (6), has the limit

$$
\widetilde{\sigma}_{\varepsilon}^{2} \stackrel{\mathrm{P}}{\rightarrow} \sigma_{\varepsilon}^{2} \frac{\int_{-c_{\alpha}}^{c_{\alpha}} \varepsilon^{2} f(\varepsilon) d \varepsilon}{\int_{-c_{\alpha}}^{c_{\alpha}} f(\varepsilon) d \varepsilon}=V\left(\varepsilon|| \varepsilon \mid<c_{\alpha}\right)
$$

For the normal distribution, $f(\varepsilon)=\phi(\varepsilon)$, we find the expression:

$$
\frac{\int_{-c_{\alpha}}^{c_{\alpha}} \varepsilon^{2} f(\varepsilon) d \varepsilon}{\int_{-c_{\alpha}}^{c_{\alpha}} f(\varepsilon) d \varepsilon}=1-\frac{2 c_{\alpha} \phi\left(c_{\alpha}\right)}{1-\alpha}
$$

Theorem 3 Let $y_{t}=\mu+\sigma_{\varepsilon} \varepsilon_{t}, t=1, \ldots, T$ be i.i.d., where $\varepsilon_{t}$ has symmetric continuous density $f($.$) with mean zero, variance one, and E\left[\varepsilon_{t}^{8}\right]<\infty$. Let $T=\sum_{j=1}^{m} T_{j}$, and assume that $T_{j} / T \rightarrow \lambda_{j}$, where $0<\lambda_{j}<1$, with $\sum_{j=1}^{m} \lambda_{j}=1$, then the limit distribution of the estimator $\tilde{\mu}$, see (17), is given by:

$$
T^{1 / 2}(\tilde{\mu}-\mu) \stackrel{\mathrm{D}}{\rightarrow} \mathrm{N}\left[0, \sigma_{\varepsilon}^{2} \sigma_{\mu}^{2}\right]
$$


where

$$
\begin{aligned}
\sigma_{\mu}^{2}= & \left(\int_{-c_{\alpha}}^{c_{\alpha}} f(\varepsilon) d \varepsilon\right)^{-2} \\
& \times\left[\int_{-c_{\alpha}}^{c_{\alpha}} \varepsilon^{2} f(\varepsilon) d \varepsilon\left(1+4 c_{\alpha} f\left(c_{\alpha}\right)\right)+\sum_{j=1}^{m} \lambda_{j}\left[\sum_{k \neq j} \frac{\lambda_{k}}{1-\lambda_{k}}\right]^{2}\left(2 c_{\alpha} f\left(c_{\alpha}\right)\right)\right]^{2}
\end{aligned}
$$

If in particular $T_{1}=\cdots=T_{m}$, then $\sum_{j=1}^{m} \lambda_{j}\left[\sum_{k \neq j} \frac{\lambda_{k}}{1-\lambda_{k}}\right]^{2}=1$.

The results of this paper are generalized to autoregressive time series in Johansen, $\mathrm{S}$. and Nielsen, B. "An analysis of the indicator saturation estimator as a robust regression estimator," The Methodology and Practice of Econometrics: A Festschrift in Honour of David F. Hendry, Oxford University Press, 2008. 